\title{
Melamine/epichlorohydrin prepolymers: syntheses and characterization
}

\author{
Luís M. Pedroso ${ }^{\mathrm{a}, *}$, M. Margarida C.A. Castro ${ }^{\mathrm{b}}$, Pedro Simões ${ }^{\mathrm{a}}$, António Portugal ${ }^{\mathrm{a}}$ \\ ${ }^{a}$ Department of Chemical Engineering, Faculty of Sciences and Technology, University of Coimbra, Pólo II, Pinhal de Marrocos, 3030-290 Coimbra, Portugal \\ ${ }^{\mathrm{b}}$ Department of Biochemistry, NMR Center and Center for Neuroscience and Cell Biology, University of Coimbra, Portugal
}

Received 9 November 2004; accepted 16 December 2004

Available online 19 January 2005

\begin{abstract}
The basic catalysis of melamine with epichlorohydrin gives prepolymers that can be used in the preparation of energetic materials. In this work, sodium hydroxide and triethylamine were used as catalysts and ethyleneglycol as initiator. Different reaction conditions were tested and the characterization of the products was carried out by IR and NMR spectroscopy and MS spectrometry, hydroxyl groups content, vapour pressure osmometry and elemental and thermal analysis. Epichlorohydrin reacts with the amine groups of melamine and forms lateral chains with hydroxyl and epoxide end groups, which can be used for curing purposes. The two catalysts lead to similar products, confirmed both by the structure and number of the lateral chains. The melamine/epichlorohydrin ratio was found important for the structure of the final compounds. Chlorine atoms leave the molecules during reaction due to basic catalysis. In the light of the use of the prepolymers in energetic materials, the presence of the 1,3,5-s-triazine ring and the lateral chains with end groups curable by e.g. isocyanates was accomplished with success. However, the loss of chlorine atoms limits to a certain extent their possible substitution by energetic groups.

(C) 2004 Elsevier Ltd. All rights reserved.
\end{abstract}

Keywords: Prepolymer synthesis; Melamine; Epichlorohydrin

\section{Introduction}

In 1945 Ericks [1] patented a process claiming the reaction of melamine with ethylene oxide (EO) or glycidol. Basic catalysts like $\mathrm{NaOH}, \mathrm{KOH}, \mathrm{Ca}(\mathrm{OH})_{2}$ or pyridine were used. Kaiser [2] added a polyol with at least two hydroxyl groups to produce similar compounds. Sodium and potassium methoxides were also used as catalysts, using temperatures between 150 and $200{ }^{\circ} \mathrm{C}$. Kucharski [3] studied the reaction between melamine and $\mathrm{EO}$ or propylene oxide (PO) in $N, N$-dimethylformamide (DMF), using tetrabutylammonium hydroxide (TBAH) as catalyst. Lower reaction temperatures, $70-95{ }^{\circ} \mathrm{C}$, were employed. Lubczak [4] used dimethyl sulfoxide (DMSO) as solvent. Mixtures of different products were found, revealing unequal side branches of the triazine ring.

It is well known that melamine derivatives also react with oxiranes via anionic polymerization, with the catalyts TBAH [5,6], triethylamine (TEA) [7-13], $\mathrm{KOH}, \mathrm{NaOH}$, sodium

\footnotetext{
* Corresponding author. Tel.: +351 239798770; fax: +351 239798703 .

E-mail address: miguel2@eq.uc.pt (L.M. Pedroso).
}

methoxide and potassium ethoxide [14]. DMSO, DMF and water were used as solvents. All the melamine derivatives contained hydroxyl end groups, formed by reaction of melamine with acetyl groups or formaldehyde. EO, PO or epichlorohydrin $(\mathrm{ECH})$ were used almost exclusively.

The objective of the present work is to synthesize and characterize melamine/ECH prepolymers and open way for a future investigation of their use in the field of energetic materials. The 1,3,5-s-triazine ring is present in many energetic materials, e.g. RDX (cyclo-1,3,5-trimethylene2,4,6-trinitramine) and in new materials like DNAM (4,6dinitramino-1,3,5-triazine-2(1H)-one) [15]. When chlorine is substituted by azide or other energetic groups, the melamine/ ECH prepolymers can supply energy to a formulation with the benefits of introducing the 1,3,5-s-triazine ring [15].

\section{Experimental}

\subsection{Materials and equipment}

Reagents: melamine (99\%) and epichlorohydrin (99\%) 
were obtained from Aldrich, ethylene glycol A R. from Riedel de Haen, sodium hydroxide p.a. from Fluka, triethylamine p.a. from Sigma, DMSO p.a. from Labscan and DMF (99\%) from Fluka.

FTIR spectra were obtained in a Nicolet 750 , using a Golden Gate ATR accessory from Specac, where the products were analysed as prepared. Resolution was 4 and the number of scans 64. NMR spectra were obtained at a Varian Unity 500 NMR spectrometer operating at $499.824310 \mathrm{MHz}$ and 25 and $80{ }^{\circ} \mathrm{C}$ using a $5 \mathrm{~mm}$ broad band probe. DMSO-d6 (99.99\% D) from Riedel de Haen was used as solvent. A $\mathrm{D}_{2} \mathrm{O}$ solution of DSS (3-(trimethylsilyl)-1-propanesulfonic acid sodium salt) was used as an internal concentration reference $-3 \mu \mathrm{l}$ of a $0.1502 \mathrm{M}$ solution was added to $500 \mu \mathrm{l}$ of DMSO-d6 solution of measured weight of the tested compound. The integrals of ${ }^{1} \mathrm{H}$ NMR signals were determined using the program NutsPro. Mass spectrometry analyses were made in a FISONS Instruments VG Quattro FAB + . Recording was made from $\mathrm{m} / \mathrm{z} 120$ to 1013 . Three different matrixes were used: 3nitrobenzyl alcohol, thioglycerol and hydroxyethyl disulfide.

Hydroxyl concentration was measured by the acetic anhydride in pyridine method, with $1 \mathrm{~h}$ of reaction time at $98^{\circ} \mathrm{C}$ [16]. A second method with phthalic anhydride in pyridine and imidazole as catalyst was employed [17]. Reaction time was $15 \mathrm{~min}$ at $98^{\circ} \mathrm{C}$. Elemental analysis was carried out in a Fisons Instruments EA 1108. HPLC was carried out in a Waters Action Analyzer with a Waters 431 Conductivity Detector.

Osmometry was carried out in a Gonotec Osmomat 070. Water was used as solvent, temperature was set at $60^{\circ} \mathrm{C}$ and sampling time at $4 \mathrm{~min}$. Chromatography was performed using a Polymer Laboratories EMD-960 evaporative light scattering detector. A Viscotek 270 Dual Detector and a Knauer K-2301 RI detector in parallel were also used. Water was the solvent used in both cases.

The visual inspection of the thermal events was done with the sample placed inside a glass capillary and then dried under vacuum. The capillary was then sealed and immersed in an oil bath. A stable heating rate was achieved through the use of a PID controlled heating bath and efficient stirring. Dynamic Mechanical Thermal Analysis (DMTA) experiments were made in a Polymer Laboratories DMTA MkII with a PL Temperature Programmer 706, PL Tensile Control and a Combined Head. A steel plate in a closed V shape was used to hold the powder samples. Single cantilever with $8 \mathrm{~mm}$ free sample length, displacement of $16 \mu \mathrm{m}$, frequency of $1 \mathrm{~Hz}$, heating rate of $2.0^{\circ} \mathrm{C} \mathrm{min}-1$ and a purge of dry nitrogen were used in all experiments. DSC tests were done in a Polymer Laboratories DSC model PL DSC at $10.0^{\circ} \mathrm{C} \mathrm{min}^{-1}$.

\subsection{Syntheses}

All syntheses were carried out in a 50 or $100 \mathrm{ml}$ glass reactor equipped with magnetic stirring and reflux condenser in a temperature controlled oil heating bath. Pre- determined quantities of melamine and the solvent DMF were added to the reactor. Stirring and heating were started. Initiator and ECH were then added and finally the catalyst. Table 1 presents the experimental conditions. A temperature of $100{ }^{\circ} \mathrm{C}$ and reaction time of $48 \mathrm{~h}$ were used in all syntheses.

The final products were allowed to cool to room temperature and were filtrated afterwards. A first distillation was then carried out to partially remove the solvent. The obtained products were precipitated in acetone and, after discarding the liquid, were repeatedly washed with acetone. At this stage the products were viscous liquids. An attempt to dry the products in a temperature controlled ventilated oven was unsuccessful. Even keeping them at $60{ }^{\circ} \mathrm{C}$ for several days, the presence of acetone was always observed. Satisfactory drying was only achieved keeping the products overnight in a vacuum oven at $40{ }^{\circ} \mathrm{C}$. Other non-solvents were tested, as ethyl acetate, diethyl ether, tetrahydrofuran and 1,2-dichloromethane, but acetone was the most satisfactory. Polar solvents like water and methanol dissolved the products, with the exception of MEC5. The dried products were very hygroscopic yellow solids. Again, MEC5 was the exception being a non-hygroscopic yellow liquid.

\section{Results and discussion}

\subsection{Spectroscopic analysis}

Fig. 1(a) shows the IR spectrum of MEC8 and Fig. 1(b) the IR spectra of the synthesized products in the range $1300-700 \mathrm{~cm}^{-1}$.

All products, except MEC5, show the bands corresponding to the $s$-triazine ring: $1565,1439,1425,1398,815$ and $750 \mathrm{~cm}^{-1}[18,19]$. The most informative band appears at $815 \mathrm{~cm}^{-1}$ and is due to the out-of-plane ring deformation (Fig. 1(b)). It is a medium intensity band except for MEC12, where it is more intense due to the high concentration of the triazine ring. The most intense band around $1565 \mathrm{~cm}^{-1}$ (see Fig. 1(a)) is attributed to the $\mathrm{N}-\mathrm{C}-\mathrm{N}$ bending and ring deformation [18].

The band at $3220 \mathrm{~cm}^{-1}$ identifies the hydroxyl group and is present in the spectra of all compounds. The 1090 and $1050 \mathrm{~cm}^{-1}$ bands shown in Fig. 1(b) are attributed to the C$\mathrm{O}$ stretching vibrations. MEC 12 shows a very weak band at $1050 \mathrm{~cm}^{-1}$. It is expected that MEC12 has less ether bonds than the other synthesized compounds due to the low 1:2 melamine/ECH ratio used. Hence, the band at $1050 \mathrm{~cm}^{-1}$ is attributed to stretching of $\mathrm{C}-\mathrm{O}$ from ether bonds [20] while the band at $1090 \mathrm{~cm}^{-1}$ is attributed to the stretching of $\mathrm{COH}$. The band at $1090 \mathrm{~cm}^{-1}$ is an indication that hydroxyl groups are secondary [21].

Table 2 shows the ratios of the heights of the bands at 1090 and $1050 \mathrm{~cm}^{-1}$ taking as reference the height of the band at $815 \mathrm{~cm}^{-1}$. IR spectra can be quantitatively analysed [20] 
Table 1

Reagents and respective amounts used in the syntheses of the prepolymers

\begin{tabular}{|c|c|c|c|c|c|c|}
\hline & Melamine (mmol) & $\mathrm{ECH}(\mathrm{mmol})$ & $\mathrm{EG}(\mathrm{mmol})$ & $\mathrm{NaOH}(\mathrm{mmol})$ & TEA (mmol) & $\mathrm{DMF}(\mathrm{ml})$ \\
\hline MEC3 & 39.679 & 396.36 & 39.630 & 8.5425 & & 30.0 \\
\hline MEC4 & 39.689 & 396.36 & & 8.2525 & & 30.0 \\
\hline MEC5 & & 396.36 & 39.630 & 8.2800 & & 30.0 \\
\hline MEC8 & 39.657 & 396.36 & & & 19.396 & 30.0 \\
\hline MEC12 & 39.695 & 79.272 & & & 19.396 & 30.0 \\
\hline
\end{tabular}

and this ratio compares the concentration of hydroxyl and ether groups having as reference the triazine ring. The higher ratios observed for MEC3 indicate higher concentrations of these groups when compared to the other compounds. MEC8 shows lower ratios than MEC3 or MEC4, which is attributed to the different catalyst used. MEC3 and MEC4 show higher values for the ratio 1050/ 815, while for MEC8 similar results are obtained for both ratios. When compared to TEA, $\mathrm{NaOH}$ leads to a higher ether/OH ratio. MEC12 shows low ratios and a low ether bonds content, which is attributed to the low melamine/ECH ratio in the synthesis. MEC5 has a very high content of these bonds, as can be seen in Fig. 1(b).

The bands at 2941 and $2884 \mathrm{~cm}^{-1}$ clearly show the
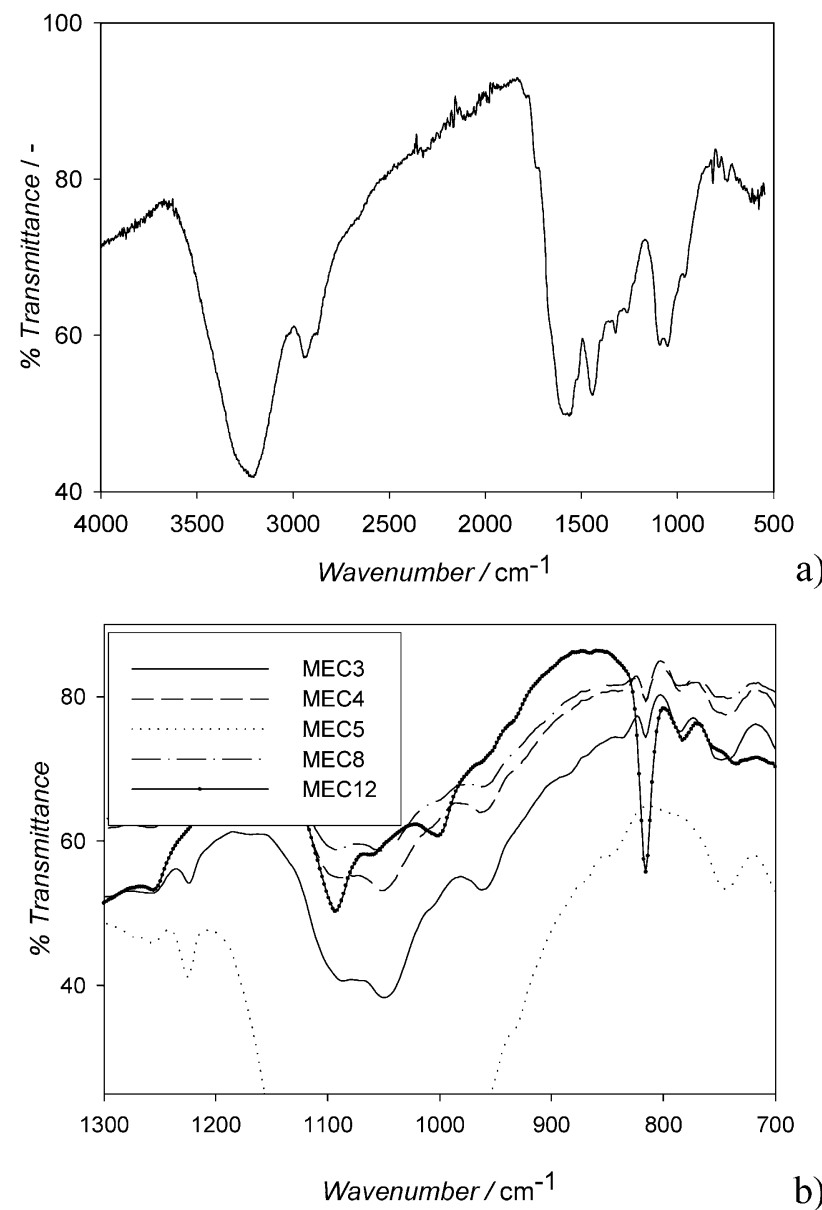

Fig. 1. IR (a) spectrum of MEC8 and (b) spectra of the prepolymers in the range $1300-700 \mathrm{~cm}^{-1}$. existence of alkyl groups in all compounds. The same can be said for the bands at 1440 and $1320 \mathrm{~cm}^{-1}$. For MEC5 these bands are observed at 1470 and $1360 \mathrm{~cm}^{-1}$.

The characteristic IR bands of amines above $3000 \mathrm{~cm}^{-1}$ cannot be seen due to the broad $\mathrm{OH}$ band that appears in the same region. The $\mathrm{NH}_{2}$ scissor vibration can be observed at $1620 \mathrm{~cm}^{-1}$ for all compounds except for MEC5. This band is broad except for MEC12, which shows a stronger and sharper band indicating that the environments of the primary amine groups in MEC12 are similar. Swing vibration mode of the amine groups is seen as a shoulder at $1007 \mathrm{~cm}^{-1}$. In all spectra except for MEC5 a shoulder is observed at around $2650 \mathrm{~cm}^{-1}$, which is also seen with the same characteristics in the melamine spectrum.

The band for the stretching vibration mode of $\mathrm{C}-\mathrm{Cl}$ group at $740 \mathrm{~cm}^{-1}$ is of moderate to weak intensity in all compounds, indicating that part of the chlorine atoms leaves the molecule during the reaction.

The IR spectrum of ECH shows a very strong band at $1265 \mathrm{~cm}^{-1}$ due to epoxide groups. In the spectra of the synthesized prepolymers, a small band is observed at this wavelength. To verify the presence of these groups, MEC8 was dissolved in aqueous $0.5 \mathrm{M} \mathrm{HCl}$ and in $0.5 \mathrm{M} \mathrm{NaOH}$ at $60{ }^{\circ} \mathrm{C}$ and the recovered products were analysed. In the first case the opening of the existent epoxide groups is expected, while when a base is added the formation of epoxides, from $-\mathrm{OH}$ and $\mathrm{CH}_{2}-\mathrm{Cl}$ end groups, is likely to occur. MEC8 treated with $\mathrm{HCl}$ showed a slightly weaker band at $1265 \mathrm{~cm}^{-1}$ when compared to untreated MEC8. This band became stronger for MEC8 treated with $\mathrm{NaOH}$. In this case, the products showed a weaker band at $750 \mathrm{~cm}^{-1}$, which confirmed that epoxide groups were formed and chlorine atoms left MEC8. With $\mathrm{HCl}$ no difference was detected for this band. These results indicate the presence of epoxide groups in MEC8. The formation of epoxides implies the deactivation of the catalyst, which was used in relatively small concentration in the syntheses. The usual

Table 2

Relative concentration of ether and hydroxyl groups taking the triazine ring as reference

\begin{tabular}{lll}
\hline & $\begin{array}{l}1090 / 815 \\
\text { (Hydroxyl) }\end{array}$ & $\begin{array}{l}1050 / 815 \\
\text { (Ether) }\end{array}$ \\
\hline MEC3 & 3.03 & 3.23 \\
MEC4 & 2.57 & 2.72 \\
MEC8 & 2.31 & 2.32 \\
MEC12 & 1.18 & 0.93 \\
\hline
\end{tabular}


$\mathrm{HCl}$ titration method for epoxides [10] was not tried because of the expected significant amine interference.

Fig. 2 shows the ${ }^{1} \mathrm{H}$ NMR spectra of DMSO-d6 solutions of MEC3, MEC4, MEC8 and MEC12.

The peaks at 2.76, 2.92 (Fig. 2(a)) and at $7.96 \mathrm{ppm}$ (not shown) are assigned to the reaction solvent DMF which could not be removed from the compounds even after some weeks in vacuum oven at $40{ }^{\circ} \mathrm{C}$ and approximately $1 \mathrm{~Pa}$. Acetone shows a peak at $2.17 \mathrm{ppm}$ [4] and the peak at $2.12 \mathrm{ppm}$ can be attributed to remaining impurities. The peak at $2.53 \mathrm{ppm}$ is due to the residual protons of the deuterated DMSO.

Fig. 2(a) shows signals at 1.18 and 1.23 ppm for MEC8 and MEC12 due to alkyl groups from the catalyst TEA. For the synthesis of MEC3 and MEC4, NaOH was used as catalyst, so these peaks were not observed. MEC8 was kept two weeks at $100{ }^{\circ} \mathrm{C}$ under vacuum. The ${ }^{1} \mathrm{H}$ NMR spectrum of the DMSO-d6 solution of the compound obtained after this treatment showed attenuated signals for DMF and no signal for acetone but the signals attributed to TEA remained unchanged (data not shown). This showed that TEA is strongly bound to the prepolymer, probably through $\mathrm{H}$ bonds. The incorporation of TEA by ionic interaction between ionic chloride and ammonium cations in the compound is not probable because it would lead to a peak in the IR spectrum around $2400-2700 \mathrm{~cm}^{-1}$. As mentioned before, the shoulder seen at $2650 \mathrm{~cm}^{-1}$ in MEC8 IR spectrum is similar to the observed in the melamine spectrum. Quaternary ammonium containing compounds show a moderate to strong peak in IR spectra [22] and thus this hypothesis is rejected. The thermal treatment of MEC8 also led to some degradation of the compound, observed by weaker signals of amine and chloroalkyl groups in the ${ }^{1} \mathrm{H}$ NMR spectrum (data not shown).

In spite of all experimental care in the preparation of the samples, water was absorbed from the surrounding atmosphere due to high hygroscopicity of the compounds, giving rise to a $\mathrm{H}_{2} \mathrm{O}$ signal at 3.3-3.5 ppm observed in Fig. 2(b). In the case of MEC 3 this signal was so intense that a saturation frequency at $3.50 \mathrm{ppm}$ was required during the acquisition of the ${ }^{1} \mathrm{H}$ NMR spectrum to suppress the $\mathrm{H}_{2} \mathrm{O}$ signal. The assignment of this signal was confirmed by adding increasing amounts of $\mathrm{H}_{2} \mathrm{O}$ to $600 \mu \mathrm{l}$ of a MEC8 solution in DMSO-d6. In this case, the $\mathrm{H}_{2} \mathrm{O}$ signal shifted from $3.39 \mathrm{ppm}$ in the DMSO-d6 solution to $4.12 \mathrm{ppm}$ after the addition of $200 \mu \mathrm{l}$ of $\mathrm{H}_{2} \mathrm{O}$ (data not shown).

MEC3, MEC4 and MEC8 show similar spectral features in this region (Fig. 2(b)). Broad peaks are observed due to the protons of the lateral chains formed from ECH. This means that these lateral chains formed on the melamine molecule have similar structures in the three compounds. The absence of specific peaks due to EG fragment in MEC3 can be attributed to a side reaction between EG and ECH, leading to the formation of low molecular weight polyepichlorohydrin removed in the purification step [23]. The peaks at $3.27-3.29,3.68$ and $4.15 \mathrm{ppm}$ for MEC8 are
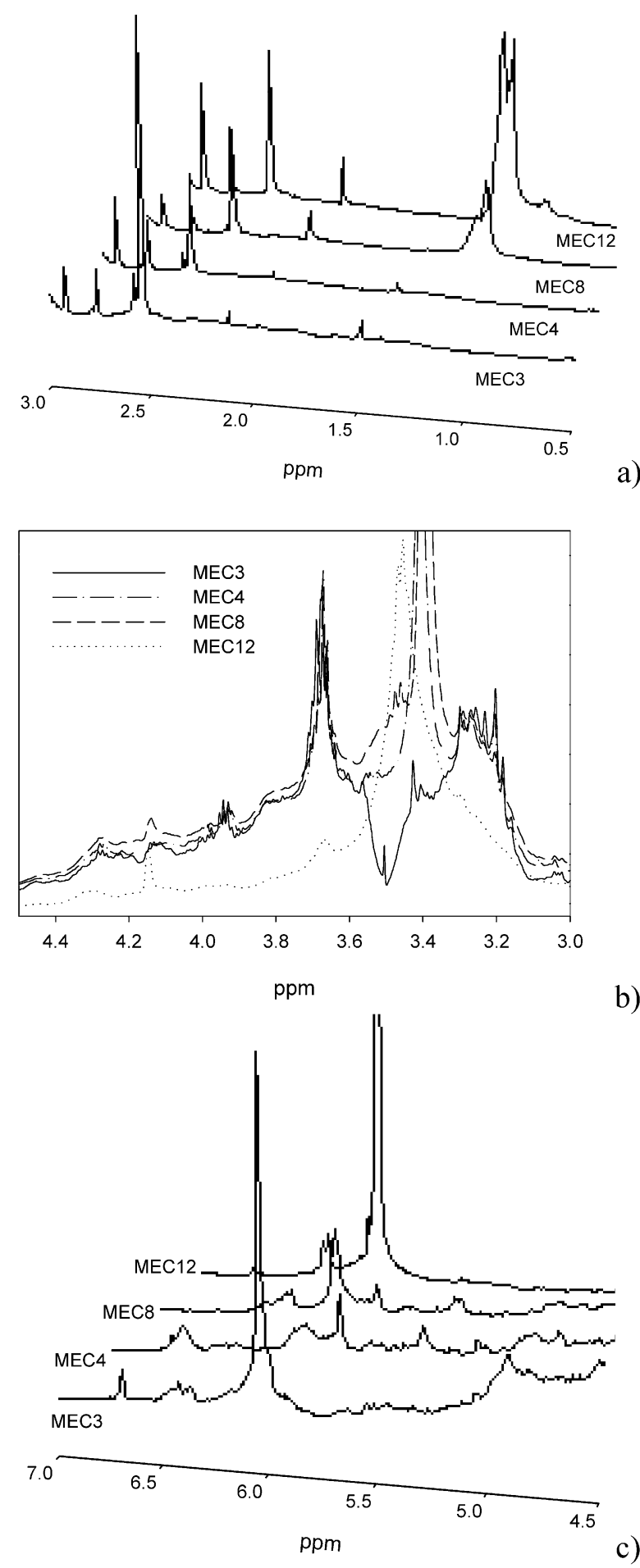

b)

Fig. 2. ${ }^{1} \mathrm{H}$ NMR spectra of DMSO-d6 solutions of MEC3, MEC4, MEC8 and MEC12 at $499.824310 \mathrm{MHz}, 25^{\circ} \mathrm{C}$ and concentrations in the range of 31 to $58 \mathrm{mg} \mathrm{ml}^{-1}$, (a) from 0.5 to $3.0 \mathrm{ppm}$, (b) 3.0 to $4.5 \mathrm{ppm}$ and (c) 4.5 to $7.0 \mathrm{ppm}$.

narrower than the ones for MEC3 and MEC4 (not very clear in Fig. 2(b)). However, the differences are not relevant. In the spectrum of MEC12, a more intense and narrower signal is observed at $4.15 \mathrm{ppm}$, the signal at $3.93 \mathrm{ppm}$ is absent, the one at $3.68 \mathrm{ppm}$ is much less intense and the signals at 
3.1-3.3 ppm are not observable, when compared to the other prepolymers. The broadness of the peaks did not allow a clear assignment of the ${ }^{1} \mathrm{H}$ NMR spectra.

The ${ }^{1} \mathrm{H}$ NMR spectrum of MEC 8 treated with $\mathrm{NaOH}$, previously analysed by IR, was obtained (data not shown). The addition of $\mathrm{NaOH}$ to MEC8 leads to the gaseous emission of $\mathrm{HCl}$. Crystals of $\mathrm{NaCl}$ were also observed in the solution. The intensity of the peak at $3.68 \mathrm{ppm}$ in the ${ }^{1} \mathrm{H}$ NMR spectrum of the treated product markedly decreased when compared to MEC8. This shows that chlorine atoms left the prepolymer, with formation of epoxide groups, which is in agreement with the IR analysis. The spectrum of MEC8 was compared to the one of the treated product in an attempt to identify the protons of the new epoxide groups. However, no significant differences could be observed from 2.4 to $3.5 \mathrm{ppm}$ because the peaks of these protons are in a region where broad peaks and the water signal appear.

Fig. 2(c) shows the ${ }^{1} \mathrm{H}$ signals of the hydroxyl groups in the region between 5.0 and $5.9 \mathrm{ppm}$ [4]. These signals disappear with the addition of $\mathrm{H}_{2} \mathrm{O}$ or $\mathrm{D}_{2} \mathrm{O}$ due to proton exchange with these solvents (data not shown). The assignment of these signals is in agreement with published data from the reaction products of melamine with formaldehyde $[3,6,9,11,24]$.

In the ${ }^{1} \mathrm{H}$ NMR spectrum of melamine in DMSO-d6, all the $\mathrm{NH}_{2}$ groups show a single signal at $6.20 \mathrm{ppm}$. The addition of oxirane shifts this signal to 5.9-6.2 ppm, and the signal from $\mathrm{NH}$ groups appears between 6.1 and $6.4 \mathrm{ppm}$ [4]. $\mathrm{NH}_{2}$ groups of $\mathrm{N}(2)$-butyl-1,3,5-triazine-2,4,6-triamine show signals at 6.04 and $6.19 \mathrm{ppm}$ and the $\mathrm{NH}$ group at $6.46 \mathrm{ppm}$ [22]. In N(2),N(2)-diethyl-1,3,5-triazine-2,4,6triamine the primary amines show a signal at $6.08 \mathrm{ppm}$ [22]. ${ }^{1} \mathrm{H}$ NMR spectra of MEC8 in DMSO-d6 were obtained before and after the addition of $\mathrm{H}_{2} \mathrm{O}$ or $\mathrm{D}_{2} \mathrm{O}$ at $80^{\circ} \mathrm{C}$ (data not shown). The peaks at $6.0-6.1 \mathrm{ppm}$ and at $6.2-6.5 \mathrm{ppm}$ attributed to $\mathrm{NH}_{2}$ and $\mathrm{NH}$ groups, respectively, vanished after the addition of the $\mathrm{H}_{2} \mathrm{O}$ or $\mathrm{D}_{2} \mathrm{O}$. This is due to the exchange of the protons between amine groups and $\mathrm{H}_{2} \mathrm{O}$ or $\mathrm{D}_{2} \mathrm{O}$, thus confirming this assignment.

To quantify the amine groups in each compound, the areas of the signals corresponding to primary amines were determined between 5.95 and $6.20 \mathrm{ppm}$, and secondary amines between 6.26 and $6.80 \mathrm{ppm}$. The appearance of ${ }^{1} \mathrm{H}$ NMR signals at different chemical shift values attributed to secondary amines shows that these protons have different chemical environments due to the different structures and stereochemistry of the lateral chains derived from ECH. For each spectrum four calculations of the integral of each signal were carried out. The numbers of moles of $\mathrm{NH}_{2}$ and $\mathrm{NH}$ groups were determined relative to the area of the DSS signal used as internal reference. Table 3 shows the results obtained. To calculate these values the molar mass of the compounds obtained by elemental analysis was used.

MEC3 shows a higher content of primary amines when compared to MEC4 or MEC8, but the content of secondary amines is lower. The lower content of secondary amines in
MEC3 when compared to MEC4 shows that EG may have a role in the reactivity of these groups. MEC4 shows lower concentration of primary amines and higher concentration of secondary amines when compared to MEC8. MEC12 shows the highest concentration of primary amines, explained by the higher melamine/ECH ratio. However, the secondary amines concentration is lower than the observed for MEC4 and MEC8. This shows that the reactivity of the secondary amines is independent of the $\mathrm{ECH}$ concentration in the solution. The calculated total number of branches is similar for MEC3, MEC4 and MEC8 and lower for MEC12. This indicates that, in the used experimental conditions, melamine/ECH ratio is more important for the substitution of amine groups of melamine than the catalyst used.

In an attempt to a better assignment of the region 3.0$4.4 \mathrm{ppm}$ of the ${ }^{1} \mathrm{H}$ NMR spectra (Fig. 2(b)), RELAY-COSY spectra of DMSO-d6 solutions of MEC4 and MEC8 were obtained. Fig. 3 shows the MEC4 2D spectrum.

Taking into account the most intense cross peaks, the following correlations can be made: the signal at $4.28 \mathrm{ppm}$ is coupled with the one at $3.93 \mathrm{ppm}$. The latter is coupled with the signals at 3.68 and $5.86 \mathrm{ppm}$, which are attributed to chloroalkyl and hydroxyl groups, respectively. Coupling between the signals at 4.14 and $3.46 \mathrm{ppm}$ and between this one and the signal at $3.28 \mathrm{ppm}$ are also observed. The signals at 3.46 and $3.28 \mathrm{ppm}$ show no other couplings, indicating that they correspond to protons from epoxide groups. The relatively low field where these signals appear is attributed to the presence of the ring [22]. Coupling between the signal at $4.14 \mathrm{ppm}$ with the signal at $6.37 \mathrm{ppm}$ shows that these $\mathrm{CH}_{2}$ protons are bound to the nitrogen atom of melamine. The weak coupling of the signal at $4.14 \mathrm{ppm}$ with a $\mathrm{N}-\mathrm{H}$ proton bound to an ether or hydroxyl group at $5.42 \mathrm{ppm}$ indicates that this proton is involved in one $\mathrm{H}$ bond and explains the broad region where the peaks due to secondary amines are observed. The attribution of the signal at $4.14 \mathrm{ppm}$ was confirmed by a more intense peak in the 1D spectrum of MEC12 when compared with the other peaks of the 1D spectra of the other compounds. In fact, shorter lateral chains are present in MEC12.

These correlations observed in the 2D spectrum support the structures proposed in Fig. 4(a) and (b). The nitrogen from melamine can be bound to a proton or to another lateral chain. The proximity of lateral chains can be observed by

Table 3

Quantification of primary and secondary amine groups by ${ }^{1} \mathrm{H}$ NMR

\begin{tabular}{llll}
\hline & $\begin{array}{l}\mathrm{NH}_{2} \\
\mathrm{~mol} \mathrm{NH}_{2} / \mathrm{mol}\end{array}$ & $\begin{array}{l}\mathrm{NH} \\
\mathrm{mol} \mathrm{NH} / \mathrm{mol}\end{array}$ & No. of branches \\
\hline MEC3 & $0.44 \pm 0.02$ & $0.21 \pm 0.07$ & 4.9 \\
MEC4 & $0.31 \pm 0.05$ & $0.59 \pm 0.11$ & 4.8 \\
MEC8 & $0.38 \pm 0.04$ & $0.44 \pm 0.06$ & 4.8 \\
MEC12 & $0.88 \pm 0.03$ & $0.29 \pm 0.03$ & 4.0 \\
\hline
\end{tabular}

Results are expressed in number of moles of $\mathrm{NH}_{2}$ or $\mathrm{NH}$ per mole of compound. 


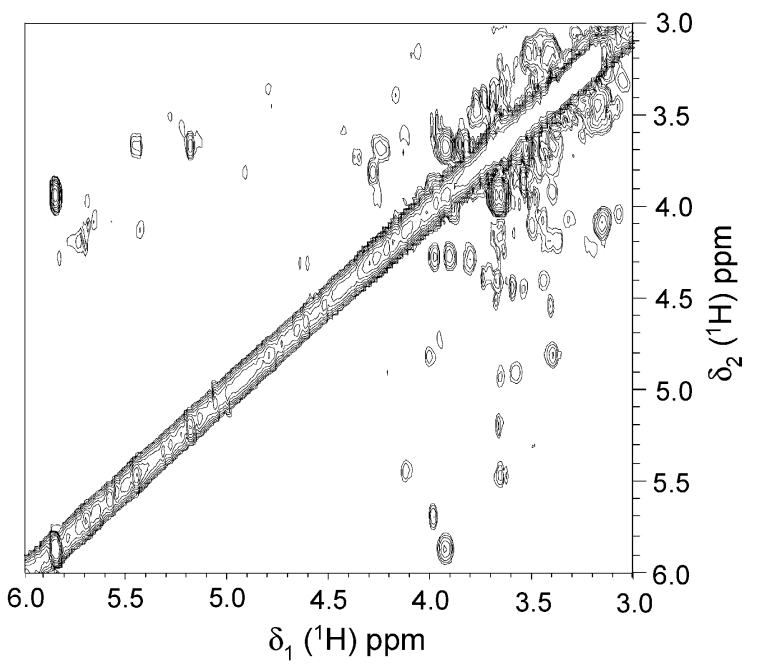

Fig. 3. RELAY-COSY spectrum of a MEC4 DMSO-d6 solution with a concentration of $54.4 \mathrm{mg} \mathrm{ml}^{-1}$, at $499.824310 \mathrm{MHz}$ and $25^{\circ} \mathrm{C}$, in the $3.0-$ $6.0 \mathrm{ppm}$ region.

some weak couplings, e.g. 5.45 with 3.66 and 3.46 with $3.68 \mathrm{ppm}$.

The lateral chains can be composed by the addition of more than one ECH molecule, leading to the structure in Fig. 4(c), which is supported by the couplings between the
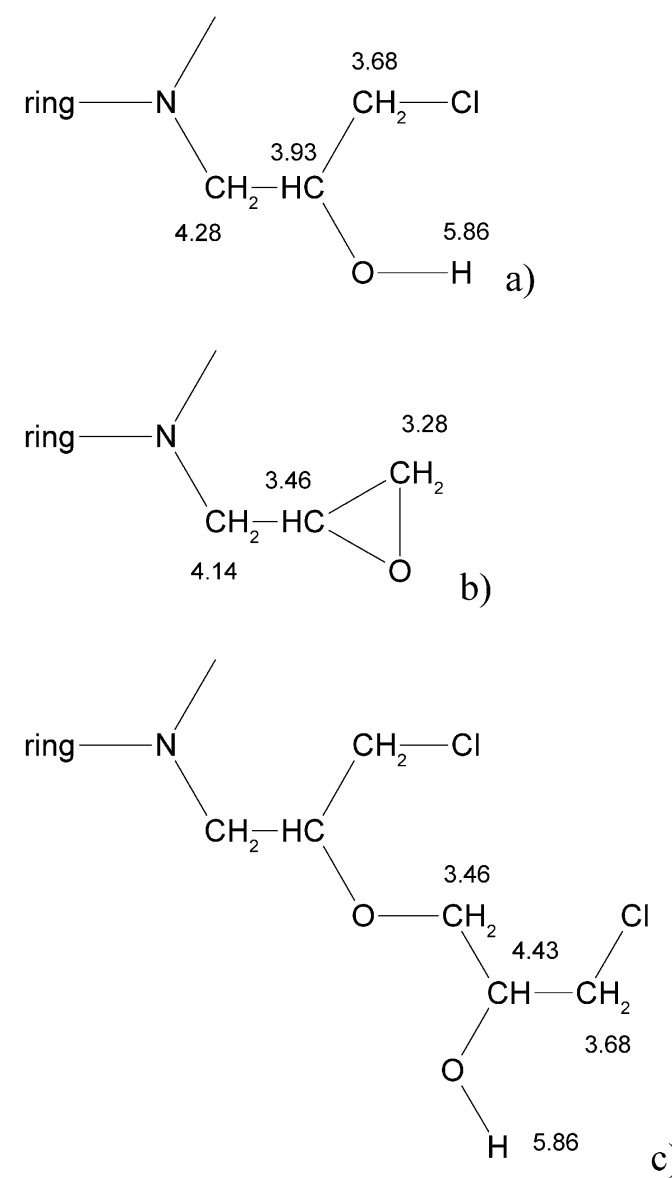

Fig. 4. Proposed structure for MEC4 based on RELAY-COSY spectrum. protons corresponding to signals at 3.46 and at $4.43 \mathrm{ppm}$ and from these ones with the protons of $\mathrm{CH}_{2} \mathrm{Cl}$ at $3.68 \mathrm{ppm}$. However, these are weaker than those observed for the protons in Fig. 4(a) and (b).

MEC8 2D spectrum (data not shown) shows the same spectral feature of the one of MEC4 and leads to the structures proposed in Fig. 4, confirming the similarity of these two compounds. Only minor remarks can be added by observation of MEC8 2D spectrum. A strong coupling is observed between the signals at 1.18 and $1.23 \mathrm{ppm}$ from TEA and a signal at 3.4-3.5 ppm attributed to $\mathrm{H}_{2} \mathrm{O}$, thus indicating interaction between TEA and water in the solution. The spectrum also shows a coupling between the primary and the secondary amines, probably through the ring.

A HMQC experiment was carried out with a DMSO-d6 solution of MEC8 (data not shown). The presence of TEA is confirmed by the signals at 11.4 and $47.9 \mathrm{ppm}$ in the ${ }^{13} \mathrm{C}$ spectrum [22]. The protons with signal at $3.46 \mathrm{ppm}$ in the ${ }^{1} \mathrm{H}$ spectrum are coupled with the carbons at $50.3 \mathrm{ppm}$. By comparison with $N(2), N(2)$-diethyl-1,3,5-triazine-2,4,6-triamine [22], the carbons of the $\mathrm{N}-\mathrm{C}$ bonds between melamine and the lateral chains have signals at 62.4 and $73.5 \mathrm{ppm}$ for secondary amines. These peaks are coupled with the ${ }^{1} \mathrm{H}$ signal at $4.14 \mathrm{ppm}$. The carbons bound to chlorine atoms show a ${ }^{13} \mathrm{C}$ signal at $50.3 \mathrm{ppm}$, which is coupled with the ${ }^{1} \mathrm{H}$ signal at $3.68 \mathrm{ppm}$. The ${ }^{13} \mathrm{C}$ spectrum also indicates that some $\mathrm{C}=\mathrm{C}$ bonds may be present, most probably at the end of the chains [25].

MEC8 and MEC12 were analysed by mass spectrometry. Analyses with three different matrixes allowed us to reject the peaks from fragments of the matrixes or of reactions of the matrixes with the compounds [26]. The identified peaks for MEC12 are $m / z$ (relative intensity) $424(0.5), 393(0.4)$, 351 (1), 295 (3), 228 (9), 210 (5), 194 (24), 176 (14), 158 (100), 144 (15), 128 (11) and 127 (24). Other peaks with lower intensities appear in the spectra. MEC12 probably is a mixture of different molecules but there are no obvious molecular ions in the spectra. Melamine-formaldehyde resins also do not show a molecular peak in $\mathrm{FAB}+$ analysis [27]. The peak at $194 \mathrm{Da}$ has a chlorine atom due to the height of the peak at $196 \mathrm{Da}$, which is about $1 / 3$ of the first. The same can be said for the peak at $176 \mathrm{Da}$. An attempt to attribute the peak at $158 \mathrm{Da}$ to a fragment with the melamine molecule or part of it was made. That was impossible without breaking the ring structure. According to the literature, the compounds $N(2), N(2)$-diallylmelamine, $N(2)$-butyl-melamine and $N(2)$-diethyl-melamine do not show ring break with EI ionization [22]. As FAB + is weaker than EI, we can conclude that the peak at $158 \mathrm{Da}$ should be attributed to a lateral chain fragment.

The identified peaks for MEC8 are $\mathrm{m} / \mathrm{z}$ (relative intensity) 351 (1), 314 (3), 295 (5), 266 (5), 252 (6), 228 (28), 210 (9), 194 (15), 186 (8), 181 (8), 176 (16), 172(11), 158 (100), 152 (8), 144 (9), 140 (13), 130 (9), 128 (10) and 127 (11). More peaks and an increased complexity were 
observed in the mass spectra of MEC 8 . Above $\mathrm{m} / \mathrm{z} 351$ there is a tail of low intensity peaks. Below 351 more fragments were observed. However, the most important peaks are present in both spectra, indicating structural similarities between MEC8 and MEC12. A clear assignment of the positive ions formed requires more detailed data, e.g. ESIMS, thus no attempt of identification was made.

\subsection{Determination of hydroxyl groups content}

Table 4 shows the results of the hydroxyl groups content measurements by two methods. Due to amine interference in the acetylation reaction, as confirmed by the partial acetylation of melamine alone, Siggia's method [28] for determining the amine concentration in mixtures of $\mathrm{OH}$ and amines was tried. It follows a procedure of two steps: in the first step the acetylation of the hydroxyl and amine groups is carried out. In the second step $\mathrm{NaOH}$ is added and the esthers previously formed from hydroxyl groups undergo a saponification reaction. It is referenced that amides do not react in this second stage [28]. The amount of $\mathrm{NaOH}$ consumed in the reaction is used to determine the hydroxyl concentration in the sample and the amine concentration can be calculated from subtraction. The two acetylation methods were used, both followed by saponification. Results are shown in Table 4.

The two acetylation methods gave slightly different results. In spite of the use of a catalyst, the acetylation reaction was less extent with the phthalic anhydride/imidazole method. The phthalic anhydride molecule is larger than the acetic anhydride and stereochemical hindrance may occur. Longer reaction times did not change the results.

The saponification method gave results that are twice higher than those from the acetylation methods giving a clear indication that some side reaction must have occurred. After the saponification step, the molecule has a terminal hydroxyl group, with a chlorine atom in beta position. As the medium is strongly basic, the chlorine atom can react with another $\mathrm{NaOH}$ and the adjacent carbon links to the $\mathrm{O}$ atom, giving a terminal closed epoxide ring and $\mathrm{NaCl}$ and water as products. This reaction explains the results obtained and allow us to say that acetylation results are valid for the determination of $\mathrm{OH}$ content.

\subsection{Elemental analysis and molar mass}

Table 5 shows the elemental analysis of the products.

Table 4

Results of acetylation and saponification of MEC8

\begin{tabular}{lcc}
\hline & Acetic anhydride (\%) & $\begin{array}{l}\text { Phthalic anhydride/ } \\
\text { imidazol }(\%)\end{array}$ \\
\hline $\begin{array}{l}\text { OH+NH from acety- } \\
\text { lation }\end{array}$ & $8.55 \pm 0.67$ & $7.07 \pm 1.20$ \\
$\begin{array}{l}\text { OH-esthers saponifi- } \\
\text { cation }\end{array}$ & $13.96 \pm 0.26$ & $14.23 \pm 0.64$ \\
\hline
\end{tabular}

The compounds are expected to have the elements $\mathrm{C}, \mathrm{H}$, $\mathrm{N}, \mathrm{Cl}$ and $\mathrm{O}$. Some deviations were found in the measurements, which can be attributed to the very high hygroscopicity of the compounds. Chlorine content measured by HPLC was compared to coulometric titration results and no significant differences were found.

The presence of the triazine ring and lateral amine groups is given by the nitrogen content. MEC5 has no melamine in its composition and some solvent contamination has occurred. MEC12 shows the highest $\mathrm{N}$ content which is due to the high melamine/ECH ratio used. Oxygen, which comes from $\mathrm{ECH}$, has a lower value.

MEC4 shows slightly higher $\mathrm{N}$ and lower $\mathrm{O}$ contents than MEC3, which indicate a higher melamine concentration in MEC4. If EG was involved in the reaction in a significant way, these differences would be higher due to the presence of the fragment $\mathrm{O}-\mathrm{CH}_{2}-\mathrm{CH}_{2}-\mathrm{O}$ in MEC3. The comparison between MEC4 and MEC8, synthesized in the presence of $\mathrm{NaOH}$ and TEA, respectively, shows a slightly higher $\mathrm{N}$ content for MEC8, but differences are again negligible and may be due to residual TEA in MEC8.

Assuming that nitrogen comes exclusively from melamine and there is only one molecule of melamine per molecule of prepolymer, the molar mass can be estimated based on elemental analysis results. These results are shown in Table 6.

The mass of the compounds was measured by a vapour pressure osmometer, being the results 238, 282 and $315 \mathrm{~g} \mathrm{~mol}^{-1}$ for MEC3, MEC4 and MEC8, respectively. An attempt to use chromatography was made but no reliable results could be obtained.

The differences between osmometry and elemental analysis results for molar mass are significant. These are attributed to aggregation of molecules in the osmometer, which lead to measured lower molar mass values. Melamine resins are prone to aggregation $[4,29]$ and the osmometer conditions can lead to this effect. Based on this assumption, the molar masses calculated from elemental analysis are more reliable.

The calculated molar mass results confirm the small differences between MEC3, MEC4 and MEC8. MEC12 shows a significant lower mass than the other compounds. Under the studied conditions, the molar mass is mainly affected by the melamine/ECH ratio.

Table 6 also shows the melamine(M)/ECH ratios in the reagents and in the prepolymers calculated from molar mass. It should be noted that the calculations for the ratio M/ $\mathrm{ECH}$ assume that chlorine atoms are still in the molecule. If some chlorine atoms leave the molecule, then this ratio is higher and a correction should be made on the basis of the chlorine content. The corrected values account for this loss of chlorine. The M/ECH ratio is always lower in the prepolymers than in the reactants except for MEC12. This is explained by the possible presence of impurities, like water, that act as initiators giving low mass poliepichlorohydrin or cyclic oligomers. For MEC12, a ratio of 1:3.74 was 
Table 5

Elemental analysis results

\begin{tabular}{llllll}
\hline & $\mathrm{C}$ & $\mathrm{H}$ & $\mathrm{N}$ & $\mathrm{O}^{\mathrm{a}}$ & $\mathrm{Cl}^{\mathrm{b}}$ \\
\hline MEC3 & $42.546 \pm 1.572$ & $7.331 \pm 0.224$ & $12.272 \pm 1.246$ & $20.868 \pm 1.623$ & $9.09 \pm 0.16$ \\
MEC4 & $41.956 \pm 2.885$ & $7.267 \pm 0.496$ & $13.273 \pm 3.307$ & $20.002 \pm 1.234$ & $8.10 \pm 0.019$ \\
MEC5 & $44.956 \pm 1.872$ & $7.812 \pm 0.367$ & $3.734 \pm 0.214$ & $26.039 \pm 1.313$ & $7.69 \pm 0.18$ \\
MEC8 & $45.222 \pm 3.595$ & $6.902 \pm 0.956$ & $13.865 \pm 2.774$ & $20.843 \pm 0.799$ & $8.29 \pm 0.019$ \\
MEC12 & $45.162 \pm 0.454$ & $7.655 \pm 0.388$ & $22.876 \pm 0.609$ & $18.689 \pm 0.833$ & $7.70 \pm 0.32$ \\
\hline
\end{tabular}

${ }^{a}$ Separate analysis (elemental analysis).

b HPLC analysis.

obtained. This result indicates that ECH reacted preferentially with the already formed oligomers, and unreacted melamine was present at the end of the reaction.

The comparison of $\mathrm{M} / \mathrm{ECH}$ values with the number of branches calculated by NMR analysis show that for MEC3, MEC4 and MEC8 the average number of ECH per branch is 1.6 to 1.7 .

The loss of chlorine atoms can be seen in the number of $\mathrm{ECH}$ molecules with chlorine (see values in M/ECH with chlorine column in Table 6).

Chlorine loss is attributed to the basic catalysts. The hydrolysis of $\mathrm{CH}_{2} \mathrm{Cl}$ groups gives $\mathrm{CH}_{2} \mathrm{OH}$, with the deactivation of the catalyst. The number of moles of $\mathrm{OH}$ groups per mole of MEC 8 can be calculated from $\% \mathrm{OH}$ and molar mass values, resulting in 2.8. This result is higher than the number of moles of ECH with chlorine, indicating that hydrolysis may have taken place. The formation of epoxide bonds from $-\mathrm{OH}$ and $\mathrm{CH}_{2} \mathrm{Cl}$ end groups occurred, as shown by NMR studies, with loss of chlorine. ECH can also behave as a trifunctional monomer [25], where the chlorine leaves the molecule and crosslinking can occur. In the initiation, after opening of the epoxide group of $\mathrm{ECH}$, an intramolecular attack of the negative alkoxide ion to the carbon attached to chlorine atom gives glycidol. Glycidol is known to be more reactive than ECH in these conditions [25] and its presence can explain in part the high solubility of the prepolymers in water. The reaction of the negative alkoxide ion with the carbon attached to chlorine can also be intermolecular or between different branches of a single molecule. MEC5 is the product of the homopolymerization of $\mathrm{ECH}$ with $\mathrm{EG}$ as initiator and also shows a low $\mathrm{Cl}$ content. The loss of chlorine is thus attributed to a combination of the described reactions.

\subsection{Thermal analysis}

A first approach to thermal analysis was made by visual inspection of the thermal events. MEC4 was tested using a real heating rate of $2.48{ }^{\circ} \mathrm{C} \mathrm{min}{ }^{-1}$. For MEC 8 $2.52{ }^{\circ} \mathrm{C} \mathrm{min}{ }^{-1}$ was used.

MEC4 is solid at room temperature. At $76.4{ }^{\circ} \mathrm{C}$ it starts to soften and some particles start to aggregate. At $80.5^{\circ} \mathrm{C}$ it starts to shrink and becomes semitransparent, which indicates a liquid form. At $98.8^{\circ} \mathrm{C}$ some gas bubbles start to emerge and at $110.5^{\circ} \mathrm{C}$ the sample started to come up through the capillary due to the size of the bubbles. Qualitatively, the thermal behaviour of MEC8 is similar to MEC4, with small temperature shifts. At $75^{\circ} \mathrm{C}$ it starts to soften and becomes liquid at $85.6^{\circ} \mathrm{C}$. Bubbles start to form at $112.1^{\circ} \mathrm{C}$ and the sample starts to go up through the capillary at $119.6{ }^{\circ} \mathrm{C}$. These results show that MEC8 is somehow more thermal resistant than MEC4.

In DMTA experiments, as the samples are powders, a closed V shaped steel plate support was used. Due to this support, the absolute $E^{\prime}$ values measured do not correspond to the sample, but to the combination steel plate/sample. As the steel has not any change in the temperature range used the changes in $E^{\prime}$ and $\tan \delta$ can be attributed to the sample alone. Fig. 5 shows the results for MEC 8 in the temperature range $-20-200{ }^{\circ} \mathrm{C}$.

A thermal event was observed at $-81.97^{\circ} \mathrm{C}$ in the $E^{\prime}$ curve and -85.01 in $\tan \delta$ due to a phase transition of MEC8 (data not shown). Fig. 5 shows the onset of $E^{\prime}$ at $50.96{ }^{\circ} \mathrm{C}$, which ends at $81.63{ }^{\circ} \mathrm{C}$. The tan $\delta$ curve shows a peak at $70.27^{\circ} \mathrm{C}$. This is the temperature region where the sample was observed to soften. The $E^{\prime}$ curve shows an onset at $133.35^{\circ} \mathrm{C}$ whereas the $\tan \delta$ curve shows a peak at $125.08^{\circ} \mathrm{C}$. These temperatures correspond to a major bubble formation observed by visual inspection. Melamine decomposes thermally above $300{ }^{\circ} \mathrm{C}$ [30], which means that these thermal events should be primarily caused by the lateral chains.

The DSC analysis of MEC8 is shown in Fig. 6. Despite the high masses used, the energy involved in the thermal events is very low and the behaviour observed by visual

Table 6

Molar mass and M/ECH ratio of the prepolymers

\begin{tabular}{llllll}
\hline & Molar mass $\left(\mathrm{g} \mathrm{mol}^{-1}\right)$ & M/ECH (reagents) & M/ECH (calc.) & M/ECH (corr.) & M/ECH with chlorine \\
\hline MEC3 & $1: 10$ & $1: 6.1$ & $1: 8.52$ & $1: 1.74$ \\
MEC4 & 685 & $1: 10$ & $1: 5.5$ & $1: 7.87$ & $1: 1.64$ \\
MEC8 & 633 & $1: 10$ & $1: 5.2$ & $1: 7.48$ & $1: 1.56$ \\
MEC12 & 606 & $1: 2$ & $1: 2.7$ & $1: 0.93$ \\
\hline
\end{tabular}




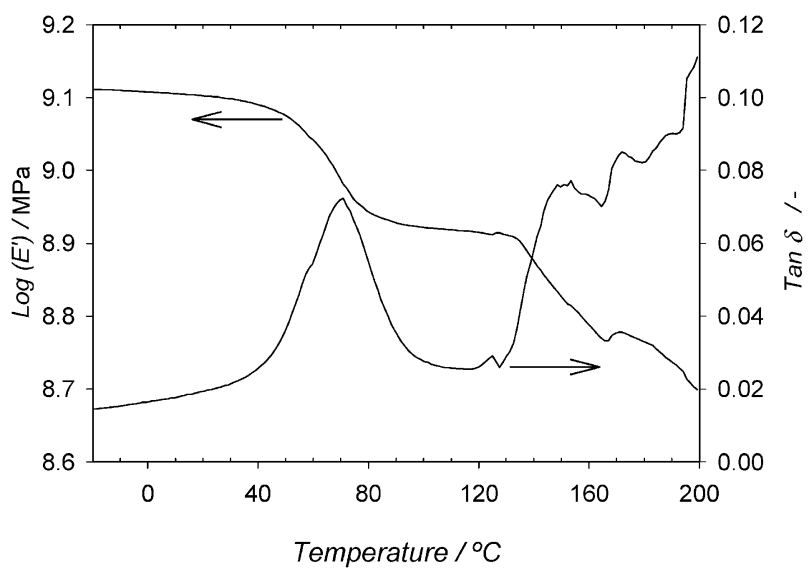

Fig. 5. DMTA analysis of MEC8 in the range -20 to $200{ }^{\circ} \mathrm{C}$.

inspection and DMTA cannot be supported by DSC. The baseline pattern from about $120{ }^{\circ} \mathrm{C}$ indicates the decomposition and gasification of the compound, as the heat capacity of the system crucible/sample is decreasing. This was visually confirmed at the end of the tests by the spongeous aspect of the residual mass in the crucible.

\section{Conclusions}

Melamine/epichlorohydrin prepolymers were synthesized using $\mathrm{NaOH}$ and TEA as catalysts. Different reaction conditions were tried and the products analysed. ECH was shown to react with melamine amine groups originating lateral chains. These have hydroxyl and epoxide end groups, which can be used in cure reactions. The structure and number of the lateral chains is similar in the compounds synthesized with TEA or $\mathrm{NaOH}$. The products synthesized with a melamine/ECH ratio of 1:10 showed 4.84.9 lateral chains per molecule, with an average of 1.6-

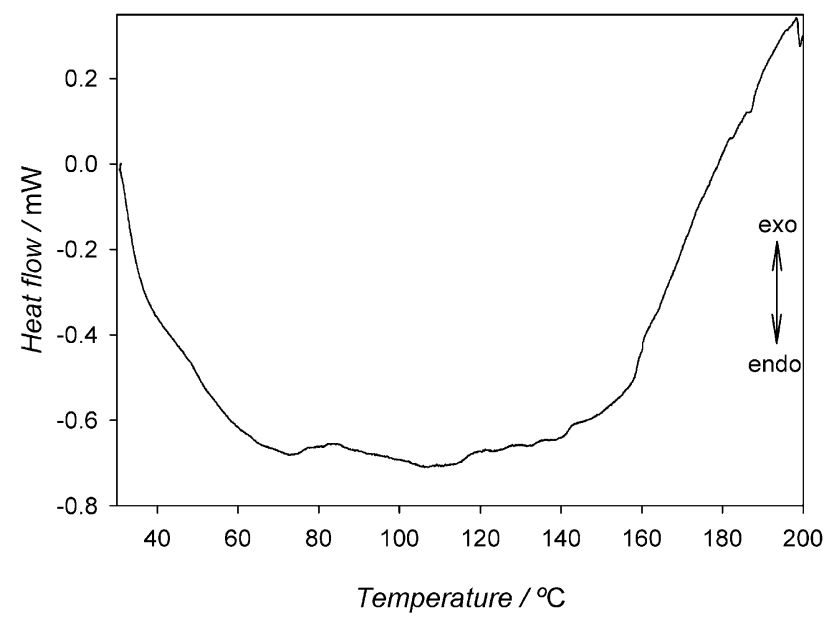

Fig. 6. DSC of MEC8.
1.7 ECH molecules incorporated in each chain. When a melamine/ECH ratio of 1:2 is used, only 4.0 lateral chains were incorporated by each melamine molecule. The structures of the lateral chains were assessed by 2D NMR spectroscopy. Molar masses are in the range from $378 \mathrm{~g} \mathrm{~mol}^{-1}$, for the lower melamine/ECH ratio, to $685 \mathrm{~g} \mathrm{~mol}^{-1}$. Chlorine atoms leave the molecules during reaction, giving products with lower concentration of chlorine than expected. The thermal analysis showed that the compounds, that are solid at room temperature, start to soften at around $51{ }^{\circ} \mathrm{C}$, with decomposition above $120{ }^{\circ} \mathrm{C}$.

In the light of the use of the prepolymers in energetic materials, the presence of the 1,3,5-s-triazine ring and the lateral chains with end groups curable by e.g. isocyanates was accomplished with success. However, the loss of chlorine atoms limits to a certain extent their possible substitution by energetic groups.

\section{References}

[1] Ericks W. US Patent 2381121, 1945.

[2] Kaiser D. US Patent 3256281, 1966.

[3] Kucharsky M, Lubczak J. Acta Polym 1991;42(4):186-9.

[4] Lubczak. J Appl Polym Sci 1995;58:559-64.

[5] Lubczak. J Appl Spectrosc 1997;51(3):438-42.

[6] Lubczak J. Acta Polym 1990;41(9):464-8.

[7] Lubczak J. Indian J Chem 1994;33B:125-31.

[8] Lubczak J. Indian J Chem 1994;33B:651-6.

[9] Lubczak J, Chmiel E. Polymery 1990;35(6):194-9.

[10] Lubczak J. J Appl Polym Sci 1997;65:2589-602.

[11] Lubczak J. J Appl Polym Sci 1997;66:423-33.

[12] Lubczak J. React Funct Polym 1998;38:51-9.

[13] Lubczak J, Mysliwiec B, Bukowski W. J Appl Polym Sci 2000;76: 824-36.

[14] Szita J., DiLeone R. US Patent 4356304, 1982.

[15] Simões P, Pedroso L, Portugal A. Propellants Explosive Pyrotech 2001;26:273-7.

[16] Ogg C, Porter W, Willits C. Ind Eng Chem, Anal Ed 1945;17:345.

[17] Carey M, Wellon S, Elder D. The Society of Plastics Industry, Inc., (SPI). Sixth International Technical/Marketing Conference, San Diego, California, USA 1983.

[18] Wang Y, Mebel A, Wu C, Chen Y, Lin C, Jiang J. J Chem Soc Faraday Trans 1997;93(19):3445-51.

[19] NIST Chemistry WebBook, NIST Standard Reference Database 69 , March 2003.

[20] Jada S. J Appl Polym Sci 1988;35:1573-92.

[21] Pasto D, Johnson C, Miller M. Experiments and techniques in organic chemistry. NJ: Prentice Hall; 1992.

[22] SDBS (2003). SDBSWeb: http://www.aist.go.jp/RIODB/SDBS/

[23] Kida T, Yokota M, Masuyama M, Nakatsuji Y, Okahara M. Síntesis 1993;5:487.

[24] Duliban J, Galina H, Lubczak J. Appl Spectros 1996;50:528.

[25] Zilkha A, Weinstein M. J Appl Polym Sci 1962;6(24):643-50.

[26] Dass C. J Mass Spectrom 1996;31:77.

[27] Chang T. Anal Chem 1994;66:3267.

[28] Siggia S, Kervenski I. Anal Chem 1951;23(1):117-8.

[29] Chang DCK, Pickett MA, Singer DF, Sorser A, Strickland DS. U.S. Patent No. 6689457, 2004

[30] Sigma-Aldrich Catalog, June 2003. 